\title{
THE FUNDAMENTAL GROUP AND THE FIRST COHOMOLOGY GROUP OF A MINIMAL SET
}

\author{
BY HSIN CHU AND MICHAEL A. GERAGHTY ${ }^{1}$
}

Communicated by G. A. Hedlund, January 16, 1963

1. Introduction. A conjecture of long standing, posed by Professor W. H. Gottschalk, is whether an $n$-sphere cannot be a minimal set under a continuous flow, for an odd $n$ greater than one. More generally, must a compact manifold which is minimal under a continuous flow have a nontrivial fundamental group. Or more generally yet, must a compact Hausdorff space which is minimal under a continuous flow have a nontrivial first integral cohomology group in the sense of Alexander-Wallace-Spanier.

Now let $X$ be a locally pathwise-connected compact Hausdorff space such that every map of $X$ into $S^{1}$ is homotopic to a constant, i.e. $\pi\left(X, S^{1}\right)=0$. Then it is shown in this paper that if $X$ is minimal under a continuous flow, $X$ must be totally minimal. The above questions then reduce to the existence of totally minimal flows.

It is further shown that for such spaces $X$ a totally minimal flow cannot be locally almost periodic. So on such spaces one cannot have a locally almost periodic minimal flow.

In particular, for a sphere or real projective space of odd dimension greater than one or for a lens space, if it is a minimal set under a continuous flow, then it is totally minimal and so it is not locally almost periodic. For terminology we refer to Gottschalk-Hedlund [4]. Incidentally, the above results constitute a partial answer to Problem 1 of [5].

2. The main theorem. In the case of compact Hausdorff spaces $X$, it is known that $\pi^{1}(X)$ the Bruschlinsky group, which as a set is just $\pi\left(X, S^{1}\right)$, is isomorphic to the first integral A-W-S cohomology group $H^{1}(X)$. (See [6].) So either of these groups being zero implies that $\pi\left(X, S^{1}\right)$ is zero and every map of $X$ to $S^{1}$ induces the zero homomorphism on $\pi_{1}(X)$. We may also derive this conclusion from the assumption that $\pi_{1}(X)$ has no factor group isomorphic with the integers.

THEOREM. Let $X$ be a compact, Hausdorff, locally pathwise-connected space such that for any map from $X$ to $S^{1}$, the induced homomorphism $f_{*}$ on $\pi_{1}(X)$ is trivial. Then if $X$ is a minimal set under a continuous flow, $X$ is totally minimal.

${ }^{1}$ This work was supported by Contract NAS8-1646 with the George C. Marshall Space Flight Center, Huntsville, Alabama. 
Proof. Let $(X, R, \pi)$ be the given transformation group. Now since $(X, R, \pi)$ is not totally minimal, there exists a closed syndetic subgroup $G$ such that for all points $b$ in $X$ the orbit closure $\mathrm{Cl}(\pi(b, G))$ is not all of $X$. Since we are concerned only with the additive structure of $R$, we may assume without loss of generality that $G$ is the subgroup $Z$ of the integers of $R$.

Let $Q_{1}$ be the relation on $X$ defined by the orbit closures of $Z$, namely $x Q_{1} y$ if $x$ is in $\mathrm{Cl}(\pi(y, Z)) . Q_{1}$ is an open and a closed relation (see [4, Chapter II]) and $X$ is normal, and under these conditions one can easily see that the quotient space $X^{*}=X / Q_{1}$ is Hausdorff. Let $p_{1}: X \rightarrow X^{*}$ be the quotient map.

Denote by $Q_{2}$ the usual "modulo one" relation on the reals $R$ and let $p_{2}: R \rightarrow S^{1}$ be the quotient map to the unit circle, denoted by the reals modulo one. We have that $\pi: X \times R \rightarrow X$ maps the relation $Q_{1} \times Q_{2}$ into the relation $Q_{1}$ and so induces a map on $(X \times R) /\left(Q_{1} \times Q_{2}\right)$. Now $Q_{1}$ and $Q_{2}$ are both open, and so we may identify $(X \times R) /\left(Q_{1} \times Q_{2}\right)$ and $\left(X / Q_{1}\right) \times\left(R / Q_{2}\right)$. Thus $\pi$ induces a continuous function $\pi^{*}$ and the diagram

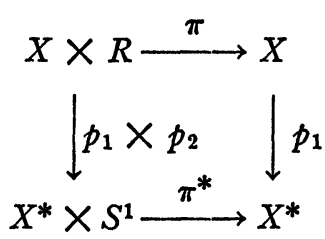

is commutative. It follows easily that $\left(X^{*}, S^{1}, \pi^{*}\right)$ is a transformation group.

For the remainder of the paper, let $b$ be any chosen base point in $X$. Define $i: R \rightarrow X \times R$ by $i(r)=(b, r)$. Consider the mapping

$$
R \stackrel{i}{\rightarrow} X \times R \stackrel{\pi}{\rightarrow} X \stackrel{p_{1}}{\rightarrow} X^{*} .
$$

By assumption, $(X, R, \pi)$ is minimal, and so $\pi \circ i(R)$ is dense in $X$ and thus $p_{1} \circ \pi \circ i(R)$ is dense in $X^{*}$.

Next consider the map

$$
R \stackrel{i}{\rightarrow} X \times R \stackrel{p_{1} \times p_{2}}{\longrightarrow} X^{*} \times S^{1} \stackrel{\pi^{*}}{\rightarrow} X^{*} .
$$

Then $\left(p_{1} \times p_{2}\right) \circ i(R)=\left\{p_{1}(b)\right\} \times S^{1}$ is homeomorphic to $S^{1}$. So $\pi^{*} \circ\left(p_{1} \times p_{2}\right) \circ i(R)$ is the continuous image in $X^{*}$ of the compact set $\left\{p_{1}(b)\right\} \times S^{1}$ and since $X^{*}$ is Hausdorff this image is compact while by the above commutative diagram, it is also dense in $X^{*}$. So

$$
\pi^{*} \circ\left(p_{1} \times p_{2}\right) \circ i(R)=p_{1} \circ r \circ i(R)=X^{*} .
$$


Now since by assumption $\mathrm{Cl}(\pi(b, Z))$ is not all of $X, X^{*}$ does not reduce to a point. In the action $\left(X^{*}, S^{1}, \pi^{*}\right)$ let $K$ be the isotropy subgroup of $p_{1}(b)$. Then $K$ is a closed and proper and so finite subgroup of $S^{1}$. Let $Z_{0}=p_{2}^{-1}(K)$ in $R$. Then the above construction of $\left(X^{*}, S^{1}, \pi^{*}\right)$ may be repeated for the subgroup $Z_{0}$ of $R$. It follows that the new isotropy subgroup will be the identity in $S^{1}$. So by proper choice of the subgroup $Z_{0}$ in $R$, we may assume that the isotropy subgroup is trivial. Again without loss of generality we may assume that $Z_{0}$ is $Z$.

We may then define $j: X^{*} \times S^{1} \rightarrow S^{1}$ by $j\left(x^{*}, s\right)=s$, and we have the diagram

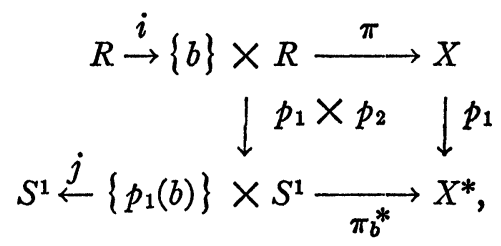

where $\pi_{b}^{*}$ is the restriction of $\pi^{*}$ to the $p_{1}(b)$-fibre. Define $g=j \circ\left(\pi_{b}^{*}\right)^{-1} \circ p_{1} \circ \pi \circ i$. Then by commutivity, $g=j \circ\left(p_{1} \times p_{2}\right) \circ i$ $=p_{2}$.

We shall need the following:

Definition. Let $f: A \rightarrow S^{1}$ be a map, where $A$ is a closed interval of $R$, one endpoint of which is zero and the other a real number $r$. Let $p_{2}: R \rightarrow S^{1}$ be the usual quotient map, with the usual orientations. Now $f$ may be lifted to a map $F: A \rightarrow R$ with $f(0)$ lifted to $F(0)$ in the interval $[0,1)$ in a unique orientation preserving fashion. Define W. N. of $f(r)=$ Winding Number of $f=F(r)-F(0)$. Note that W. N. of $p_{2}$ at $r$ is $r$, for all real $r$.

Now the key to the proof is to show that while $g$ and $p_{2}$ are identical by commutivity, their winding numbers are distinct.

Define $m=j \circ\left(\pi_{b}^{*}\right)^{-1} \circ p_{1}: X \rightarrow S^{1}$. Then by assumption, since $m_{*}\left(\pi_{1}(X)\right)=0$, the degree of $m$ is zero. Choose $0<\delta<\frac{1}{2}$, and let $\alpha$ be an index in $X$ such that for $(x, y)$ in $\alpha, d(m(x), m(y))<\delta$, where $d$ is the usual distance on the reals modulo one. Let $\beta^{2}$ be in $\alpha$, and let $V$ be a pathwise-connected neighborhood of $b$ such that $V$ is in $\beta(x)$. Then for $y$ and $z$ in $V$,

(a) there is a path $\sigma: I \rightarrow V, I=[0,1]$, where $\sigma(0)=y, \sigma(1)=z$;

(b) the winding number of the function $m \circ \sigma: I \rightarrow S^{1}$ is less than $2 \delta<1$ in absolute value.

Now let $\pi(b, r)$, for some $r>0$, be any later point of the orbit of $b$ which lies in $V$. Such exist since $X$ is compact minimal. Then $\pi \circ i$ : $R \rightarrow X \times R \rightarrow X$, restricted to the interval $[0, r]$, is a path $\mu$ in $X$ from $b$ to $\pi(b, r)$. Let $\sigma$ be a path in $V$ from $\pi(b, r)$ to $b$. 
Let $T=[0, r] \cup[0,1]$, the union space. Define $\Sigma: T \rightarrow X$ by $\Sigma=\mu \cup \sigma$. Now $S^{1}$ is a quotient space of $T$, upon identifying endpoints, and $\Sigma$ induces a map $\Sigma^{*}: S^{1} \rightarrow X$. Then

$$
m \circ \Sigma^{*}: S^{1} \rightarrow S^{1} \text {. }
$$

But this map factors through $X$. So $m \circ \Sigma^{*}$ induces a trivial map from $\pi_{1}\left(S^{1}\right)$ to $\pi_{1}\left(S^{1}\right)$.

Now the degree of $m \circ \Sigma^{*}$ is the sum of the winding numbers of $m \circ \Sigma^{*}$ restricted to the images of $[0, r]$ and $[0,1]$ with the usual orientation. But this degree must be zero. So the winding number of $m \circ \mu$ is the negative of the winding number of $m \circ \sigma$.

Then $\mid$ W. N. of $m \circ \mu(r) \mid<2 \delta<1$. But $m \circ \mu=g$ on $[0, r]$. So $\mid$ W. N. of $g(r) \mid<2 \delta<1$. Now since $X$ is compact minimal there is some $r_{0}>1$ in $R$ such that $\pi\left(b, r_{0}\right)$ is in $V$. Then

$$
\mid \text { W. N. of } g\left(r_{0}\right) \mid<1,
$$

while W. N. of $p_{2}\left(r_{0}\right)=r_{0}>1$.

The main theorem follows from this contradiction. There is an alternate proof using the covering map property (see [6]) on the above diagram.

3. Some corollaries. We need the following:

LEMMA. Let $(X, T, \pi)$ be a transformation group, where $X$ is compact, Hausdorff and $T$ is a locally compact, non-totally-disconnected, abelian group. Let $T_{0}$ be the connected component of the identity in $T$ and assume that $a$ in $X$ is such that $\pi\left(a, T_{0}\right) \neq a$. Let $X$ be minimal and locally almost periodic under $T$. Then $X$ is not totally minimal.

Proof. By Theorem 10.07 of [4], we have for any $b$ in $\pi\left(a, T_{0}\right)$, $b \neq a$, that $a$ and $b$ are distal. Denoting by $P$ the proximal relation, since $X$ is locally almost periodic we have that $P$ is a closed equivalence relation (see [3]) and the induced transformation group $\left(X / P, T, \pi^{*}\right)$ is almost periodic minimal. Also $a P \neq b P$. So by the theorem of [2], $X / P$ is not totally minimal, and so neither is $X$.

The completion of this proof was aided by a conversation with J. Auslander.

We now have the following four corollaries.

CoRollary 1. No sphere or real projective space of dimension greater than one, nor any lens space, can be a locally almost periodic minimal set under a continuous flow.

PRoof. Immediate from the main theorem and the lemma. 
Corollary 2. Let $(X, R, \pi)$ be a transformation group where $R$ is the real numbers and $X$ is compact, Hausdorff, locally pathwise-connected, minimal and not totally minimal. Then $\pi\left(X, S^{1}\right)=\pi^{1}(X) \approx H^{1}(X)$ $\neq 0$, and $\pi_{1}(X)$ must have a factor group isomorphic to the integers.

Proof. A restatement of the main theorem.

CoRollaRy 3. Let $(X, R, \pi)$ be a transformation group where $R$ is the real numbers and $X$ is a compact Hausdorff orientable manifold, minimal and not totally minimal. Then $H_{1}(X), H^{1}(X)$ and $\pi^{1}(X)$ have factor groups isomorphic to the integers.

Proof. In this case we have $H_{1}^{w}(X) \approx H_{v}^{1}(X)$ while $H_{1}(X)$ is $\pi_{1}(X)$ modulo its commutator subgroup. The corollary follows from Corollary 2 .

Corollary 4. If $G$ is a nontrivial, separable, connected, locally pathwise-connected, compact Hausdorff abelian group, then $H^{1}(G)$ is nontrivial and $\pi_{1}(G)$ has a factor group isomorphic to the integers.

Proof. Every such group is almost periodic minimal under some continuous flow. (See [1].)

\section{REFERENCES}

1. H. Anzai and S. Kakutani, Bohr compactifications of a locally compact abelian group. I, II, Proc. Imp. Acad. Tokyo 19 (1943), 476-480, 533-539.

2. Hsin Chu, On totally minimal sets, Proc. Amer. Math. Soc. 13 (1962), 457-458.

3. R. Ellis and W. H. Gottschalk, Homomorphisms of transformation groups, Trans. Amer. Math. Soc. 94 (1960), 258-271.

4. W. H. Gottschalk and G. A. Hedlund, Topological dynamics, Amer. Math. Soc. Colloq. Publ. Vol. 36, Amer. Math. Soc., Providence, R. I., 1955.

5. W. H. Gottschalk, Minimal sets, Bull. Amer. Math. Soc. 64 (1958), 336-351.

6. S. T. Hu, Homotopy theory, Academic Press, New York, 1959.

University of ALABAMa 